\title{
The Association Between Postoperative Recovery and Psychosocial Factors in Cancer Patients
}

\author{
Saho Wada ${ }^{1,2}$, Ryoichi Sadahiro ${ }^{1,2}$, Yutaka Matsuoka ${ }^{1,2,3}$, Yosuke Uchitomi ${ }^{1,3,4}$, Ken Shimizu ${ }^{1,2,3}$ \\ ${ }^{1}$ Department of Psycho-Oncology, National Cancer Center Hospital. Tokyo, Japan \\ ${ }^{2}$ Division of Health Care Research, Behavioral Sciences and Survivorship Research Group, Center for Public Health Sciences, National Cancer \\ Center Japan, Tokyo, Japan \\ ${ }^{3}$ Innovation Center for Supportive, Palliative and Psychosocial Care, National Cancer Center Hospital, Tokyo, Japan \\ ${ }^{4}$ Behavioral Sciences and Survivorship Research Group, Center for Public Health Sciences, National Cancer Center Japan, Tokyo, Japan
}

\section{Email address:}

sahowada22@gmail.com (S. Wada), rsadahir@ncc.go.jp (R. Sadahiro), yumatsuo@ncc.go.jp (Y. Matsuoka), yuchitom@ncc.go.jp (Y. Uchitomi),keshimiz@ncc.go.jp (K. Shimizu)

\section{To cite this article:}

Saho Wada, Ryoichi Sadahiro, Yutaka Matsuoka, Yosuke Uchitomi, Ken Shimizu. The Association Between Postoperative Recovery and Psychosocial Factors in Cancer Patients. Journal of Surgery. Vol. 8, No. 1, 2020, pp. 9-15. doi: 10.11648/j.js.20200801.13

Received: January 5, 2020; Accepted: January 20, 2020; Published: February 4, 2020

\begin{abstract}
Surgery is one of the main treatments component against cancer. Although the basic principle of surgical treatment of cancer is curability, safety, and functionality, early recovery after surgery is also important. While some previous studies focus on physical recovery, there are few studies regarding the perioperative period of cancer patients and their impact on postoperative recovery. This study is aim to clarify psychosocial factors that affect postoperative recovery in cancer patients. The study design is a secondary data analysis of a prospective observational cohort study whose primary purpose was to investigate whether preoperative anxiety predicted onset of postoperative delirium in cancer patients at the National Cancer Center Hospital in Japan. The primary outcome of this study was the length of postoperative hospital stay, and the secondary outcome was postoperative complications. We conducted multivariable regression analysis to determine psychosocial predictors of primary and secondary outcomes. The final analysis included 109 patients. The mean length of postoperative hospital stay was $23.4(\mathrm{SD}=12.4)$ days and the incidence rate of postoperative complications was $32.1 \%$. In multivariable regression, preoperative anxiety was positively associated with the length of postoperative hospital stay, while sex (female) and alcohol dependence showed a negative association. Cognitive function was marginally associated with postoperative complications. In conclusion, Appropriate management of preoperative anxiety contributes to early discharge after surgery in cancer patients.
\end{abstract}

Keywords: Oncology, Surgery, Recovery, Anxiety

\section{Introduction}

Surgery is one of the main treatments for cancer. A questionnaire survey of 4,054 Japanese cancer patients and their family members indicated that approximately $75 \%$ of cancer patients underwent surgery during the course of cancer treatment, and in about half of these patients, surgery was the first-line therapy [1]. Patients involved in the survey reported that they had to decide whether to undergo surgery during a stressful and relatively confusing period just after their cancer diagnosis. The survey also suggested that surgery causes not only a physical burden but also psychosocial stressors related to loss of physical function, changes in appearance, economic problems, anxiety about re-entering society, and changing social roles and relationships in both cancer patients and their family.

While the basic goals of the surgical treatment of cancer are to safely achieve cure and functionality, early postoperative recovery is also important in cancer patients because most of them need to undergo chemotherapy and/or radiotherapy after surgery. In recent years, Enhanced Recovery After Surgery (ERAS) protocols have received much attention [2-7]. ERAS is a program that highlights surgical best practices and consists of a number of evidence-based principles that support early recovery from surgery; key elements include patient/family education, achieving intestinal tract function early in the postoperative 
period, pain control, and infection control. Importantly, all of these focus primarily on physical recovery.

By contrast, few studies have focused on cancer patients' psychosocial issues in the perioperative period and their impact on postoperative recovery. Some studies, however, suggested that preoperative psychiatric symptoms reduce quality of life in cancer patients and can even worsen surgical outcomes. For example, surgical cancer resection causes anxiety that exacerbates unfavorable symptoms [8], including prolonging postoperative pain $[9,10]$. Perioperative depression and anxiety have been reported to alter endocrine and immune functions, leading to cancer progression and worsened long-term prognoses [11-16]. We previously conducted a prospective observational study in cancer patients who underwent highly invasive surgery, and showed that preoperative anxiety affected the incidence of postoperative delirium [17]. In this context, we expected that perioperative psychosocial factors would affect the postoperative recovery of cancer patients. The aim of this study was to clarify the psychosocial factors that affect postoperative recovery in cancer patients.

\section{Methods}

\subsection{Setting and Subjects}

This study was a secondary data analysis of a prospective observational cohort study that investigated the association between preoperative anxiety and postoperative delirium in cancer patients from October 2015 through April 2016 at the National Cancer Center Hospital in Japan $(\mathrm{NCCH})$. All study protocols were approved by the Institutional Review Board and the Ethics Committee of the $\mathrm{NCCH}$, and were conducted in accordance with the Declaration of Helsinki and the criteria set out in the Strengthening the Reporting of Observational Studies in Epidemiology (STROBE) checklist. The study was registered as UMIN000018980.

The inclusion criteria were 1) a clinical or pathological diagnosis of cancer, 2) scheduled tumor resection in which the predicted operation time was 6 hours or more, and 3) age 20 years or older. The exclusion criteria were 1) too physically or psychologically ill to participate, as judged by the attending physicians or researchers; 2) inability to read or understand the informed consent documents; and 3) surgery lasting less than 6 hours due to intraoperative findings.

\subsection{Measures}

\subsubsection{Demographic and Clinical Characteristics}

Demographic data, including age, sex, and socioeconomic variables (educational level, marital status, and current employment) were obtained during face-to-face interviews. Clinical characteristics (primary cancer site, time since diagnosis, and preoperative cancer treatment) and the American Society of Anesthesiology (ASA) class [18-22] were obtained from patients' medical charts.

\subsubsection{Psychological Factors}

Preoperative anxiety: Preoperative anxiety was evaluated by the Hospital Anxiety and Depression Scale-Anxiety (HADS-A) [23, 24], a self-report instrument consisting of seven items. Symptoms occurring in the previous 2 weeks are scored on a 4-point Likert scale with total scores ranging from 0 to 21 . The HADS is the most extensively validated scale for screening emotional distress in cancer patients, and its main strength in this regard is its ability to assess anxiety and depressive symptoms without referring to physical symptoms. Kugaya et al. investigated the reliability and validity of the Japanese version of the HADS in Japanese cancer patients [24]. They showed that the Cronbach's $\alpha$ of the HADS-A was 0.77 , and the correlation between test and retest scores was 0.73. The HADS-A also yielded high sensitivity and specificity with a cut-off point of $7 / 8$. Therefore, in this study we defined a HADS-A score of 8 or more as indicative of clinical anxiety.

Postoperative delirium: Postoperative delirium was assessed by a trained psychiatrist or psychologist using the Diagnostic and Statistical Manual of Mental Disorders, Fifth Edition (DSM-5) [25]; this is one of the most rigorous methods to determine whether delirium is present or not. We clinically demonstrated and discussed our findings before this study, with a final determination made once there was satisfactory agreement between the researchers and the principal investigator (K. S). During the course of the study, the researchers assessed a sample of patients simultaneously and independently to check the inter-rater reliability of the DSM-5 (20 times for each researcher), and the kappa value was 0.90 .

Other factors: Cognitive function assessed using the Mini-Mental State Examination (MMSE) [26, 19, 20, 22], alcohol dependence measured using the Cut Down, Annoyed, Guilty, Eye-opener (CAGE) questionnaire [26, 22], and history of smoking (including current smoking) were obtained during face-to-face interviews.

\subsubsection{Surgical Factors}

The surgical factors were duration of surgery, intraoperative blood loss, incidence of postoperative delirium, postoperative complications assessed by the Clavien-Dindo classification (including postoperative delirium), and the length of postoperative hospital stay. As in studies on ERAS [27-30], we considered postoperative complications and the length of postoperative hospital stay to be indicators of postoperative recovery,.

\subsection{Procedure}

We consecutively recruited patients who were admitted to the NCCH to undergo highly invasive surgery. Demographic and medical data, cognitive functioning (MMSE), history of smoking, and the CAGE questionnaire were evaluated on admission by trained research assistants using patient interviews and chart reviews.

We evaluated preoperative anxiety using the HADS-A in the afternoon of the day before surgery. Then we assessed 
whether delirium, as defined by the DSM-5, developed during the 5-day postoperative period. We used this 5-day duration because several previous studies suggested that delirium usually appeared between 2 and 3 days postoperatively [31-33]. Other postoperative complications were assessed up to 30 days after surgery or until discharge.

\subsection{Statistical Analysis}

Statistical analyses were performed using IBM SPSS Statistics 26. Descriptive statistics were used to describe baseline characteristics and outcome measures. We considered age, MMSE score, duration of operation, amount of blood loss and length of postoperative hospital stay to be continuous variables, while other factors were treated as nominal categorical variables. Two multivariable regression analyses was performed to assess psychosocial factors associated with postoperative recovery. First, the length of postoperative hospital stay was used as the dependent variable and the following variables were included as independent variables: age, sex, type of surgery, alcohol dependence (CAGE $\geq 2$ ), MMSE, ASA, history of smoking, and preoperative anxiety (HADS-A $\geq 8$ ). Postoperative delirium was also added to the multivariable model because a strong association with preoperative anxiety was revealed in our previous study [11]. Multicollinearity was tested, and variables with a Pearson correlation of 0.6 or greater were excluded. Second, postoperative complications, including postoperative delirium (diagnosis of delirium with DSM-5 and Clavien-Dindo classification $\geq 2$ ), were entered into the model as the dependent variable, and the aforementioned variables, excluding postoperative delirium, were input as independent variables.

\section{Results}

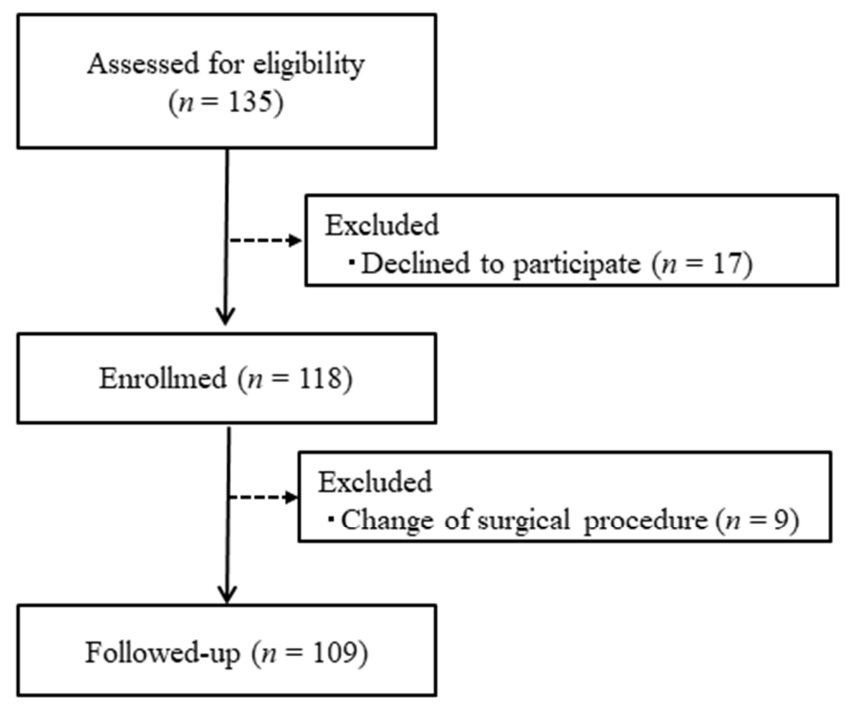

Figure 1. Flow chart outlining derivation of the final sample.

A flow chart outlining the selection of the study participants is shown in Figure 1. Of 135 consecutive patients who were eligible, $118(87.4 \%)$ were enrolled. Nine were excluded after surgery because the operations were changed to less invasive surgeries, such as intestinal bypass surgery and exploratory laparotomy. The final analysis included 109 patients. There was no significant difference in age, sex, primary cancer site, or preoperative treatment between patients who refused to participate and those who were enrolled.

Table 1. Demographic and patient characteristics.

\begin{tabular}{|c|c|c|}
\hline \multirow[b]{2}{*}{ Characteristics } & \multicolumn{2}{|c|}{ Total Population $(n=109)$} \\
\hline & $n$ & $\%$ \\
\hline $\begin{array}{l}\text { Age } \\
\text { mean (SD), years } \\
\text { Sex }\end{array}$ & $66.0(10.0)$ & \\
\hline $\begin{array}{l}\text { Male } \\
\text { Education }\end{array}$ & 76 & 69.7 \\
\hline $\begin{array}{l}\text { College graduate or higher } \\
\text { Marital status }\end{array}$ & 60 & 55.0 \\
\hline $\begin{array}{l}\text { Married or has a partner } \\
\text { Current employment }\end{array}$ & 89 & 81.7 \\
\hline $\begin{array}{l}\text { Employed } \\
\text { MMSE }\end{array}$ & 53 & 48.6 \\
\hline $\begin{array}{l}\text { mean }(\mathrm{SD}) \\
\text { CAGE score }\end{array}$ & $27.4(2.5)$ & \\
\hline ( $\geq 2$ “yes” responses) & 16 & 15.4 \\
\hline $\begin{array}{l}\text { History of smoking } \\
\text { Preoperative anxiety } \\
\text { (HADS-A } \geq 8 \text { ) }\end{array}$ & 73 & 67.0 \\
\hline
\end{tabular}

SD, standard deviation; MMSE, Mini Mental State Examination; CAGE, the Cut Down, Annoyed, Guilty, Eye-opener; HADS, Hospital Anxiety and Depression Scale.

Table 2. Medical characteristics.

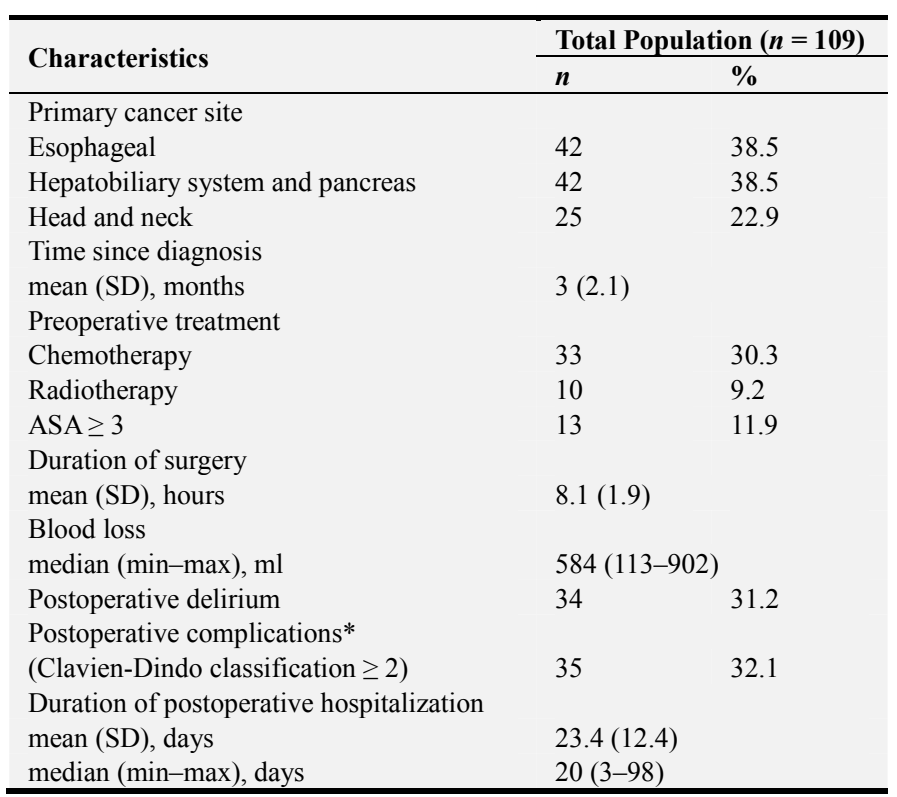

SD, standard deviation; ASA, American Society of Anesthesiologists class. *Including postoperative delirium with a Clavien-Dindo classification of more than two.

Tables 1 and 2 show data on patient demographics and medical characteristics. In the total population $(N=109)$, participants averaged 66 years of age, and the majority were male $(69.7 \%)$ and had a partner $(81.7 \%)$. Most of them 
$(88.1 \%)$ had good physical status $(\mathrm{ASA} \leq 2)$. The mean MMSE score was $27.4(\mathrm{SD}=2.5)$. The primary cancer types were esophageal cancer, $n=42(38.5 \%)$; hepatobiliary and pancreatic cancer, $n=42(38.5 \%)$; and head and neck cancer, $n=25(22.9 \%)$. Nineteen patients $(17 \%)$ had preoperative anxiety. The incidence rate of postoperative complications was $32.1 \%$.

Figure 2 shows a histogram of the length of postoperative hospital stay; the mean was $23.4(\mathrm{SD}=12.4)$ days, with a median of 20 (3-98) days.

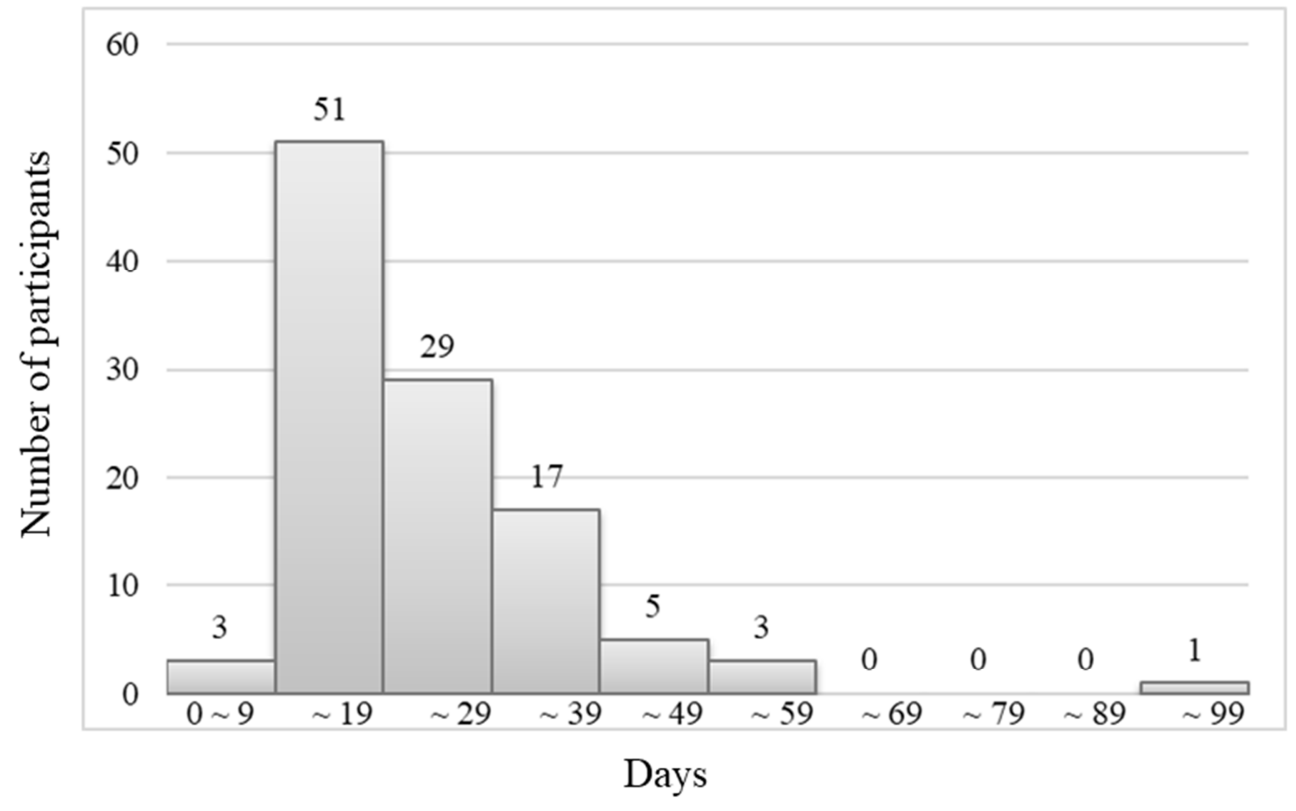

Figure 2. Histogram of the length of postoperative hospital stay.

Table 3. Multiple linear regression analysis of the length of postoperative hospital stay.

\begin{tabular}{|c|c|c|c|c|c|c|}
\hline \multirow{2}{*}{$\begin{array}{l}\text { Variable } \\
\text { Ageț }\end{array}$} & \multirow{2}{*}{$\begin{array}{l}\text { B } \\
-0.047\end{array}$} & \multirow{2}{*}{$\begin{array}{l}\boldsymbol{\beta} \\
-0.038\end{array}$} & \multirow{2}{*}{$\begin{array}{l}\boldsymbol{p} \text { value } \\
0.737\end{array}$} & \multicolumn{3}{|c|}{ 95\% Confidence Interval } \\
\hline & & & & -0.323 & - & 0.229 \\
\hline Sex (Female) ${ }^{*}$ & -7.728 & -0.287 & 0.012 & -13.697 & - & -1.760 \\
\hline $\mathrm{ASA}^{*}$ & 1.581 & 0.062 & 0.539 & -3.511 & - & -6.673 \\
\hline MMSE $\ddagger$ & -0.057 & -0.012 & 0.916 & -1.133 & - & 1.019 \\
\hline Alcohol dependence $^{*}$ & -9.024 & -0.264 & 0.009 & -15.769 & - & -2.278 \\
\hline History of smoking ${ }^{*}$ & 0.810 & 0.031 & 0.765 & -4.547 & - & -6.167 \\
\hline Preoperative anxiety ${ }^{*}$ & 6.662 & 0.209 & 0.046 & 0.131 & - & 13.193 \\
\hline Type of surgery ${ }^{*}$ & 1.999 & 0.129 & 0.193 & -0.515 & - & 2.513 \\
\hline Postoperative delirium ${ }^{*}$ & 2.645 & 0.101 & 0.320 & -2.611 & - & 7.902 \\
\hline
\end{tabular}

ASA, American Society of Anesthesiologists class; MMSE, Mini-Mental State Examination.

* Categorical variables.

$\ddagger$ Continuous variables.

Table 3 shows the results of the multiple linear regression analysis in which length of postoperative hospital stay was the dependent variable. The analysis showed that preoperative anxiety was positively associated with the length of postoperative hospital stay, while sex (female) and alcohol dependence were negatively associated. The adjusted $R^{2}$ showed that the regression model explained $9 \%$ of the variance in the length of postoperative hospital stay.

Table 4 shows the results of the multiple logistic regression analysis investigating the association between postoperative complications and psychosocial variables. The result of the goodness-of-fit test was $p=0.064$, indicating that the goodness of fit of the regression equation was low. Although the result is as a reference, there was an association between the MMSE and postoperative complications.
Table 4. Multiple logistic regression analysis of postoperative complications.

\begin{tabular}{lll}
\hline & $\begin{array}{l}\text { Odd ratio (95\% Confidence } \\
\text { Interval) }\end{array}$ & $\boldsymbol{p}$ value \\
\hline Age $\ddagger^{\dagger}$ & $1.000(0.943-1.060)$ & 0.995 \\
ASA $^{*}$ & $0.547(0.166-1.809)$ & 0.323 \\
MMSE $\ddagger^{*}$ & $1.641(0.612-4.404)$ & 0.325 \\
Alcohol dependence $^{*}$ & $0.764(0.623-0.945)$ & 0.013 \\
History of smoking $^{*}$ & $0.348(0.088-1.385)$ & 0.134 \\
Preoperative anxiety $^{*}$ & $1.417(0.496-4.045)$ & 0.515 \\
Type of surgery $^{*}$ & $1.128(0.306-4.157)$ & 0.857 \\
\hline
\end{tabular}

ASA, American Society of Anesthesiologists class; MMSE, Mini-Mental State Examination.

* Categorical variables.

$\ddagger$ Continuous variables. 


\section{Discussion}

In the multiple regression analysis, we showed that sex, alcohol dependence, and preoperative anxiety were associated with length of postoperative hospital stay in cancer patients. Our findings supported our expectation that perioperative psychosocial factors would affect postoperative recovery. Of the aforementioned variables, preoperative anxiety and alcohol dependence are potential targets for preoperative intervention. Anxiety in particular is an important target symptom because it prolongs postoperative pain $[9,10]$, impairs decision-making ability [34], and reduces treatment compliance [8, 12]. Previous studies suggested that inflammatory cytokines contribute to anxiety, though the mechanism is unclear [35-37]. We believe that anxiety may reflect a patient's physical vulnerability and consequently affect postoperative outcomes.

Contrary to our prediction, alcohol dependence was negatively associated with the length of postoperative hospital stay. One possible reason is that CAGE is a self-reported screening test, and it may not have accurately reflected the amount of alcohol consumption. Previous studies suggested that individuals often deny to report or minimize the quantity and frequency of drinking, and the accuracy of self-reporting varies depending on the interview method used [38-40]. We should have used other tools or biomarkers, such as gamma-glutamyl transferase, that more accurately reflect the amount of alcohol. Another reason for the unexpected negative association between alcohol dependence and length of hospital stay is that other physical and medical factors that were not addressed in this study affected CAGE.

The MMSE was associated with postoperative complications. Many studies have revealed that cognitive function is a risk factor for postoperative delirium $[26,19,20$, 22]. Because we included postoperative delirium as a postoperative complication, the MMSE results may have influenced the outcome.

In this study, we used the length of postoperative hospital stay and postoperative complications as indicators of postoperative recovery. Several recovery assessment tools that have been developed recently contain functional, physical, and psychological domains [41, 42]. We need to accurately define postoperative recovery and assess it using a variety of approaches, not only to reduce complications and sequelae associated with surgical treatment, but also to support the ability of cancer patients to re-enter society and to improve their quality of life.

This study has several limitations. First, the sample size was small and only a limited number of dependent variables could be entered into the regression analyses. Therefore, the models did not include several surgical and physical factors that were previously shown to affect postoperative recovery, including performance status, operation time, amount of blood loss, and the period during which patients could not eat. The second is the selection bias. This study included only patients with esophageal, hepatobiliary, pancreatic, or head and neck cancer, which may not be representative of patients in the general surgical population. For example, the demographics of patients may differ from those of general surgery because of certain risk factors for these cancers, such as smoking, alcohol, and hepatitis virus. In addition, patients in this study underwent high invasive surgery that could affect postoperative recovery. Therefore, we should be careful when generalizing our results to patients who have undergone other surgeries. Third, approximately $10 \%$ of enrolled patients could not be followed up due to changes in their surgical procedures, which reduced our sample size. Finally, due to our study design which was a secondary analysis, our results do not clearly explain why perioperative psychosocial factors predicted postoperative study.

\section{Conclusion}

Our results provide useful information on postoperative recovery in cancer patients. Appropriate management of preoperative anxiety could contribute to cancer patients' early discharge after surgery. Our findings also suggest that we can use preoperative cognitive function as an indicator to identify high-risk postoperative complications. This is the first study to investigate the association between postoperative recovery and psychosocial factors. Further well-designed studies are needed to substantiate these results.

\section{Conflict of Interest Disclosures}

Dr. Wada has received a research grant from the Health Care Science Institute. Dr. Sadahiro has received Sasakawa Foundation Butterfield awards (B108). Dr. Matsuoka has received a Grant-in-Aid for Scientific Research (B) from the Japan Society for the Promotion of Science (17H04253), National Cancer Center Research and Development Fund (30-A-17), and a research grant from SENSHIN Medical Research Foundation; and has received speaker's honoraria from Suntory, Pfizer, Mochida, Eli Lilly, Morinaga Milk and NTT Data. Dr. Uchitomi received a Grant-in-Aid for Scientific Research (B) (19H03878) and Grant-in-Aid for Exploratory Research (17K19867) from the Japan Society for the Promotion of Science, the National Cancer Center Research and Development Fund (27-A-3), Research for Promotion of Cancer Control Programmes (H29-political-general), and Japan Agency for Medical Research and development, AMED. Dr. Shimizu has received a grant from TSUMURA \& CO.

\section{Acknowledgements and Grant Support}

The authors express special gratitude to the physicians and nurses of the departments that cooperated with this study. This work was supported by The Health Care Science Institute Research Grant.

\section{References}

[1] Yamaguchi K. Gan to Mukiatta 4,054nin no Koe. (in Japanese). Survey on worries and burdens of cancer survivors. 2016. 
[2] Cerantola Y, Valerio M, Persson B, Jichlinski P, Ljungqvist $O$, Hubner M, et al. Guidelines for perioperative care after radical cystectomy for bladder cancer: Enhanced Recovery After Surgery (ERAS $((\mathrm{R})))$ society recommendations. Clin Nutr. 2013 Dec; 32 (6): 879-87.

[3] Connor S, Cross A, Sakowska M, Linscott D, Woods J. Effects of introducing an enhanced recovery after surgery programme for patients undergoing open hepatic resection. HPB (Oxford). 2013 Apr; 15 (4): 294-301.

[4] Dunne DF, Yip VS, Jones RP, McChesney EA, Lythgoe DT, Psarelli EE, et al. Enhanced recovery in the resection of colorectal liver metastases. J Surg Oncol. 2014 Aug; 110 (2): 197-202.

[5] Stowers MD, Lemanu DP, Hill AG. Health economics in Enhanced Recovery After Surgery programs. Can J Anaesth. $2015 \mathrm{Feb} ; 62$ (2): 219-30.

[6] Dort JC, Farwell DG, Findlay M, Huber GF, Kerr P, Shea-Budgell MA, et al. Optimal Perioperative Care in Major Head and Neck Cancer Surgery With Free Flap Reconstruction: A Consensus Review and Recommendations From the Enhanced Recovery After Surgery Society. JAMA Otolaryngol Head Neck Surg. 2017 Mar 1; 143 (3): 292-303.

[7] Ljungqvist O, Scott M, Fearon KC. Enhanced Recovery After Surgery: A Review. JAMA Surg. 2017 Mar 1; 152 (3): 292-98.

[8] Koizumi A, Matsushima E, Mochizuki Y, Omura K, Amagasa T. Changes in the psychological characteristics of oral cancer patients in the perioperative period: a quantitative evaluation. J Med Dent Sci. 2013 Mar 1; 60 (1): 41-53.

[9] Aigner CJ, Hernandez M, Koyyalagunta L, Novy D. The association of presurgery psychological symptoms with postsurgery pain among cancer patients receiving implantable devices for pain management. Support Care Cancer. 2014 Sep; 22 (9): 2323-8.

[10] Schreiber KL, Kehlet H, Belfer I, Edwards RR. Predicting, preventing and managing persistent pain after breast cancer surgery: the importance of psychosocial factors. Pain Manag. 2014; 4 (6): 445-59.

[11] Spiegel D, Giese-Davis J. Depression and cancer: mechanisms and disease progression. Biol Psychiatry. 2003 Aug 1; 54 (3): 269-82.

[12] Horowitz M, Neeman E, Sharon E, Ben-Eliyahu S. Exploiting the critical perioperative period to improve long-term cancer outcomes. Nat Rev Clin Oncol. 2015 Apr; 12 (4): 213-26.

[13] Bouchard LC, Antoni MH, Blomberg BB, Stagl JM, Gudenkauf LM, Jutagir DR, et al. Postsurgical Depressive Symptoms and Proinflammatory Cytokine Elevations in Women Undergoing Primary Treatment for Breast Cancer. Psychosom Med. 2016 Jan; 78 (1): 26-37.

[14] Ghoneim MM, O'Hara MW. Depression and postoperative complications: an overview. BMC Surg. 2016 Feb 02; 16: 5.

[15] Hodes GE, Menard C, Russo SJ. Integrating Interleukin-6 into depression diagnosis and treatment. Neurobiol Stress. 2016 Oct; 4: $15-22$.

[16] Andersen BL, Goyal NG, Westbrook TD, Bishop B, Carson WE, 3rd. Trajectories of Stress, Depressive Symptoms, and Immunity in Cancer Survivors: Diagnosis to 5 Years. Clin Cancer Res. 2017 Jan 01; 23 (1): 52-61.
[17] Wada S, Inoguchi H, Sadahiro R, Matsuoka YJ, Uchitomi Y, Sato T, et al. Preoperative Anxiety as a Predictor of Delirium in Cancer Patients: A Prospective Observational Cohort Study. World J Surg. 2018 Aug 20.

[18] Leung JM, Sands LP, Mullen EA, Wang Y, Vaurio L. Are preoperative depressive symptoms associated with postoperative delirium in geriatric surgical patients? J Gerontol A Biol Sci Med Sci. 2005 Dec; 60 (12): 1563-8.

[19] Kalisvaart KJ, Vreeswijk R, de Jonghe JF, van der Ploeg T, van Gool WA, Eikelenboom P. Risk factors and prediction of postoperative delirium in elderly hip-surgery patients: implementation and validation of a medical risk factor model. J Am Geriatr Soc. United States: 2006. Vol. 5, p. 817-22.

[20] Smith PJ, Attix DK, Weldon BC, Greene NH, Monk TG. Executive function and depression as independent risk factors for postoperative delirium. Anesthesiology. 2009 Apr; 110 (4): 781-7.

[21] Serafim RB, Dutra MF, Saddy F, Tura B, de Castro JE, Villarinho LC, et al. Delirium in postoperative nonventilated intensive care patients: risk factors and outcomes. Ann Intensive Care. 2012; 2 (1): 51.

[22] Shah S, Weed HG, He X, Agrawal A, Ozer E, Schuller DE. Alcohol-related predictors of delirium after major head and neck cancer surgery. Arch Otolaryngol Head Neck Surg. 2012 Mar; 138 (3): 266-71.

[23] Snaith RP, Zigmond AS. The hospital anxiety and depression scale. Br Med J (Clin Res Ed). 1986 Feb 1; 292 (6516): 344.

[24] Kugaya A, Akechi T, Okuyama T, Okamura H, Uchitomi Y. Screening for psychological distress in Japanese cancer patients. Jpn J Clin Oncol. 1998 May; 28 (5): 333-8.

[25] Takahashi S, Oono y. Desk reference to the diagnostic criteria from DSM-5. 2014.

[26] Litaker D, Locala J, Franco K, Bronson DL, Tannous Z. Preoperative risk factors for postoperative delirium. Gen Hosp Psychiatry. 2001 Mar-Apr; 23 (2): 84-9.

[27] Barber EL, Van Le L. Enhanced Recovery Pathways in Gynecology and Gynecologic Oncology. Obstet Gynecol Surv. 2015 Dec; 70 (12): 780-92.

[28] Gemmill EH, Humes DJ, Catton JA. Systematic review of enhanced recovery after gastro-oesophageal cancer surgery. Ann R Coll Surg Engl. 2015 Apr; 97 (3): 173-9.

[29] Li M, Zhang W, Jiang L, Yang J, Yan L. Fast track for open hepatectomy: A systemic review and meta-analysis. Int J Surg. 2016 Dec; 36 (Pt A): 81-89.

[30] Wang L, Zhu C, Ma X, Shen K, Li H, Hu Y, et al. Impact of enhanced recovery program on patients with esophageal cancer in comparison with traditional care. Support Care Cancer. 2017 Feb; 25 (2): 381-89.

[31] Yoshimura Y, Kubo S, Shirata K, Hirohashi K, Tanaka H, Shuto $\mathrm{T}$, et al. Risk factors for postoperative delirium after liver resection for hepatocellular carcinoma. World J Surg. 2004 Oct; 28 (10): 982-6.

[32] Takeuchi M, Takeuchi H, Fujisawa D, Miyajima K, Yoshimura $\mathrm{K}$, Hashiguchi $\mathrm{S}$, et al. Incidence and risk factors of postoperative delirium in patients with esophageal cancer. Ann Surg Oncol. 2012 Nov; 19 (12): 3963-70. 
[33] Hempenius L, Slaets JP, van Asselt DZ, Schukking J, de Bock $\mathrm{GH}$, Wiggers $\mathrm{T}$, et al. Interventions to prevent postoperative delirium in elderly cancer patients should be targeted at those undergoing nonsuperficial surgery with special attention to the cognitive impaired patients. Eur J Surg Oncol. 2015 Jan; 41 (1): 28-33.

[34] El Jawahri A, Traeger L, Park E, Greer J, Pirl W, Lennes I, et al. Associations among prognostic understanding, quality of life, and mood in patients with advanced cancer.

[35] Oliveira Miranda D, Soares de Lima TA, Ribeiro Azevedo L, Feres O, Ribeiro da Rocha JJ, Pereira-da-Silva G. Proinflammatory cytokines correlate with depression and anxiety in colorectal cancer patients. Biomed Res Int. 2014.

[36] Furtado M, Katzman MA. Neuroinflammatory pathways in anxiety, posttraumatic stress, and obsessive compulsive disorders. Psychiatry Res. 2015 Sep 30; 229 (1-2): 37-48.

[37] Murphy TM, O'Donovan A, Mullins N, O'Farrelly C, McCann A, Malone K. Anxiety is associated with higher levels of global
DNA methylation and altered expression of epigenetic and interleukin-6 genes. Psychiatr Genet. 2015 Apr; 25 (2): 71-8.

[38] Steinweg DL, Worth H. Alcoholism: the keys to the CAGE. Am J Med. 1993 May; 94 (5): 520-23.

[39] Frank D, DeBenedetti AF, Volk RJ, Williams EC, Kivlahan DR, Bradley KA. Effectiveness of the AUDIT-C as a screening test for alcohol misuse in three race/ethnic groups. J Gen Intern Med. 2008 Jun; 23 (6): 781-7.

[40] Freeman WM, Vrana KE. Future prospects for biomarkers of alcohol consumption and alcohol-induced disorders. Alcohol Clin Exp Res. 2010 Jun; 34 (6): 946-54.

[41] Bowyer A, Royse CF. The future of postoperative quality of recovery assessment: multidimensional, dichotomous, and directed to individualize care to patients after surgery. Curr Opin Anaesthesiol. 2016 Dec; 29 (6): 683-90.

[42] Bowyer AJ, Royse CF. Postoperative recovery and outcomes--what are we measuring and for whom? Anaesthesia. 2016 Jan; 71 Suppl 1: 72-7. 